\title{
Emission Spectroscopy for the Temperature Measurement of Salt-Water Ice during Hypervelocity Impact
}

Jon Tandy (1), Vassilia Spathis (2), Penny Wozniakiewicz (2) and Luke Alesbrook (2)

(1) School of Human Sciences, London Metropolitan University, London, UK, N7 8DB (j.tandy@londonmet.ac.uk)

(2) School of Physical Sciences, Ingram Building, University of Kent, Canterbury, UK, CT2 7NH

\section{Introduction}

The intense, short-lived, self-luminous plume (or 'impact flash') produced from a hypervelocity impact is frequently observed on the lunar surface [1-4]. Laboratory measurements of these impact flashes (e.g. Figure 1) can also be acquired to determine parameters such as the target and impactor composition, or size/mass of the impactor [5-9]. Previous work has shown that the emission intensity from hypervelocity impacts tends to increase at higher impact velocities [10-15]. The relative intensity of atomic and molecular emission lines/bands originating from both projectile and target materials can also be used to determine approximate temperatures reached during impact [16-20].

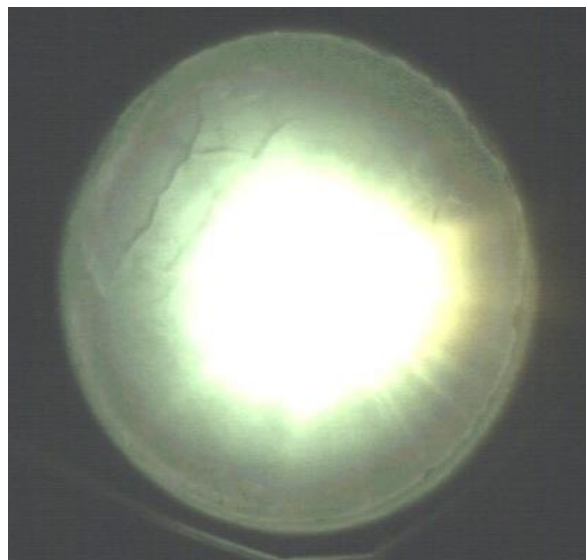

Figure 1: Photograph of a hypervelocity impact flash from a $3 \mathrm{~mm}$ aluminium projectile impacting a water ice target at $4.51 \mathrm{~km} \mathrm{~s}^{-1}$.

Current understanding of icy Solar System bodies, such as Europa and Enceladus, suggests they may contain favourable environmental conditions to synthesise biologically significant molecules such as amino acids, fatty acids, sugars and heterocyclic bases.
Ground-breaking impact experiments [21-24] have demonstrated that complex organic molecules can be formed during hypervelocity impact events that are ubiquitous throughout the Solar System. Consequently, laboratory impact flash measurements from icy targets can be utilised to constrain the temperatures required for the shock-synthesis of these biologically important species. This preliminary study utilises emission spectra for the temperature measurement of impacted salt-water ice using different projectile speeds and materials.

\section{Experimental}

The University of Kent two-stage light-gas gun (LGG) [25] was used to horizontally accelerate a $3 \mathrm{~mm}$ aluminium or $4.3 \mathrm{~mm}$ Nylon projectile into salt-water ice targets composed of $20 \mathrm{~g} \mathrm{NaCl}$ in $1 \mathrm{~L}$ of deionized water inside a sterilised 100 $\mathrm{mm}$ diameter, stainless steel container with their surfaces aligned at $90^{\circ}$ to the shot line. Specific impact parameters for each experiment are summarised in Table 1.

\begin{tabular}{c|c|c} 
Shot ID & $\begin{array}{c}\text { Projectile } \\
\text { Material }\end{array}$ & $\begin{array}{c}\text { Impact Speed } \\
/ \mathbf{~ k m ~ s}^{-1}\end{array}$ \\
\hline 1 & $\begin{array}{c}3 \mathrm{~mm} 7075 \\
\text { Aluminium }\end{array}$ & 6.03 \\
2 & $\begin{array}{c}3 \mathrm{~mm} 7075 \\
\text { Aluminium }\end{array}$ & 6.29 \\
3 & $4.3 \mathrm{~mm}$ Nylon 6 & 6.90 \\
4 & $4.3 \mathrm{~mm}$ Nylon 6 & 5.99
\end{tabular}

Table 1: Experimental parameters for impact flash measurements from salt-water ice targets.

The target mixture was frozen to $-120{ }^{\circ} \mathrm{C}$, with the temperature increasing to approximately $-50{ }^{\circ} \mathrm{C}$ during the evacuation process of the LGG target chamber (to 50 mbar), prior to firing. 
A manual focus, $50 \mathrm{~mm}$, F1.2, Nikon NIKKOR lens was aligned with the front viewport of the LGG target chamber and focused onto the end of a $0.5 \mathrm{~mm}$ internal diameter core of a fibre optic cable connected to an Ocean Insight Red Tide USB-650 spectrometer to record the impact flash spectrum.

\section{Results and Conclusions}

Figure 2 shows an example impact flash spectrum recorded using this methodology, with $\mathrm{Na}, \mathrm{Al}$ and $\mathrm{Zn}$ atomic emission lines and $\mathrm{AlO}$ molecular bands clearly visible. The Al, AlO and $\mathrm{Zn}$ emission originate from the $7075 \mathrm{Al}$ projectile (containing $\sim 6 \% \mathrm{Zn}$ ). The relative intensities of the averaged $\mathrm{Na} 589 \mathrm{~nm}$ and $819 \mathrm{~nm}$ doublet emission lines originating from the target material were used to determine approximate peak temperatures for each impact experiment using a Boltzmann distribution calculation as outlined by Unnikrishnan et al. [26].

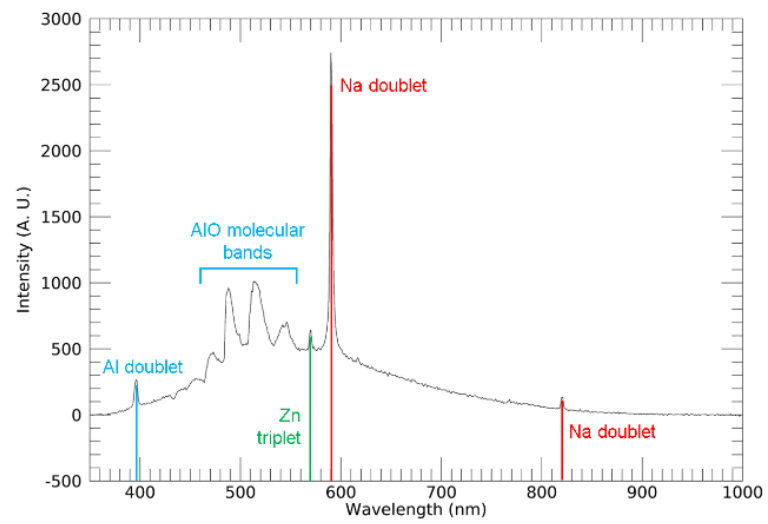

Figure 2: Impact flash emission spectrum from a $3 \mathrm{~mm}$ Al projectile impacting a salt-water ice target $6.03 \mathrm{~km} \mathrm{~s}^{-1}$. Labelled $\mathrm{Na}$ atomic lines were used for peak temperature determination.

All determined temperatures using this method were between $3000 \mathrm{~K}$ and $3420 \mathrm{~K}$. Furthermore, shots 1 and 4, with similar impacts speeds, showed a small temperature difference of $140 \mathrm{~K}$ despite the distinctly different projectile material properties. This suggests that the calculated peak temperature is derived primarily from the target material. This observation may be a result of the method only utilising $\mathrm{Na}$ emission lines originating from the salt-water ice. Future work will constrain the calculated temperatures more precisely using spectra from shots at a wider range of impact speeds. These experiments should also provide an approximate correlation of impact energy and resulting temperature to be ascertained. Additional measurements with improved spectral resolution will further increase the precision of the determined temperatures and allow complimentary calculations using atomic emission lines originating from the projectile (e.g. the Al doublet at $\sim 395 \mathrm{~nm}$ ).

\section{Acknowledgements}

The authors thank the STFC for financially supporting this work and Mark Price for IDL support.

\section{References}

[1] Uesugi K. (1993) Adv. Astronaut. Sci., 84, 607. [2] Yanagisawa, M. \& N. Kisaichi (2002) Icarus, 159, 31. [3] Bozanos A.Z. et al. (2018) A\&A, 612, A76. [4] Madiedo J.M. et al. (2018) MNRAS, 480, 5010. [5] Eichhorn G. (1976) Planet. Space Sci., 24, 771. [6] Lawrence R.J. et al. (2006) IJIE, 33, 353. [7] Ernst C.M. \& Schultz P.H. (2007) Icarus, 190, 334. [8] Goel A. et al. (2015) IJIE, 84, 54. [9] Avdellidou C. \& Vaubaillon J. (2019) MNRAS, 484, 5212. [10] Gehring J.W. \& Warnica R.L. (1963) 6th HVIS Conference Proceedings. [11] Jean B. \& Rollins T.L. (1970) AIAA J., 8, 1742. [12] Eichhorn G. (1975) Planet. Space Sci., 23, 1519. [13] Burchell M. et al. (1996) Icarus, 122, 359. [14] Ernst C.M. \& Schultz P.H. (2002) Lunar Planet. Sci., XXXIII, Abstract \#1782. [15] Sugita et al. (2003) J. Geo. Res. 108(E12), 5140. [16] Tsembelis K. et al. (2008) IJIE, 35, 1368. [17] Yafei H. et al. (2019) IJIE, 125, 173. [18] Tandy J.D. et al. (2014) J. Appl. Phys., 116. [19] Mihaly J.M. et al. (2015) J. Appl. Mech., 82. [20] Schultz P.H. \& Eberhardy C.A. (2015) Icarus, 248, 448. [21] Martins Z. et al. Nature. Geo., 6, 1045, (2013). [22] Sugahara H. \& Mimura K. (2014) Geochem. J., 48, 51. [23] Sugahara H. \& Mimura K. (2015), Icarus, 257, 103. [24] Umeda Y. et al. (2016), J. Biol. Phys., 42, 177. [25] Burchell M.J. et al. (1999) Meas. Sci. Technol., 10, 41. [26] Unnikrishnan et al. (2010) Pramana J. Phys., 74, 6. 